\section{(2) OPEN ACCESS}

\title{
Characteristics and outcomes of patients with suspected heart failure referred in line with National Institute for Health and Care Excellence guidance
}

\author{
Alice Zheng (1) , ${ }^{1,2}$ Elena Cowan (1) ,' Lukas Mach, ${ }^{2}$ Robert D Adam, ${ }^{2}$ Kaushik Guha, \\ Peter James Cowburn $\odot{ }^{2}{ }^{2}$ Paul Haydock, ${ }^{2}$ Paul R Kalra, ${ }^{1}$ Andrew Flett, ${ }^{2}$ \\ Geraint Morton ${ }^{1}$
}

- Additional material is published online only. To view please visit the journal online (http://dx.doi.org/10.1136/ heartjnl-2019-316511).

${ }^{1}$ Cardiology, Portsmouth Hospitals NHS Trust, Portsmouth, UK

${ }^{2}$ Cardiology, University Hospital Southampton NHS Foundation Trust, Southampton, UK

\section{Correspondence to} Dr Alice Zheng, Cardiology, Portsmouth Hospitals NHS Trust, Portsmouth PO6 3LY, UK; alicewzheng@gmail.com

Received 17 January 2020 Revised 29 May 2020 Accepted 2 June 2020 Published Online First 20 July 2020

\section{Linked}

- http://dx.doi.org/10.1136/ heartjnl-2020-317450

Check for updates

(C) Author(s) (or their employer(s)) 2020. Re-use permitted under CC BY-NC. No commercial re-use. See rights and permissions. Published by BMJ.

To cite: Zheng A, Cowan E, Mach L, et al. Heart

2020;106:1579-1585.

\section{ABSTRACT}

Objective To describe the population, heart failure (HF) diagnosis rate, and 1-year hospitalisation and mortality of patients with suspected $\mathrm{HF}$ and elevated $\mathrm{N}$-terminal pro B-type natriuretic peptide (NTproBNP) investigated according to UK National Institute for Health and Care Excellence (NICE) guidelines.

Methods NICE recommends patients with suspected $H F$, based on clinical presentation and elevated NTproBNP, are referred for specialist assessment and echocardiography. Patients should be seen within 2 weeks when NTproBNP is $>2000 \mathrm{pg} / \mathrm{mL}$ (2-week pathway: 2WP) or within 6 weeks when NTproBNP is $400-2000 \mathrm{pg} / \mathrm{mL}$ (6-week pathway: 6WP). This is a retrospective, multicentre, observational study of consecutive patients with suspected HF referred from primary care between 2014 and 2016 to dedicated secondary care HF clinics based on the NICE 2WP and 6WP. Data were obtained from hospital records and episode statistics. Mortality and hospitalisation rates were calculated 1 year from NTproBNP measurement. Results 1271 patients (median age 80; IQR 73-85) were assessed, $680(53 \%)$ of whom were female. 667 $(53 \%)$ were referred on the 2WP and $604(47 \%)$ on the 6WP. 698 (55\%) were diagnosed with HF (369 HF with reduced ejection fraction) and $566(45 \%)$ as not HF (NHF). 1-year mortality was $10 \%(n=129)$ and hospitalisation was $33 \%(n=413)$. Patients on the 2WP had higher mortality and hospitalisation rates than those on the $6 \mathrm{WP}, 14 \%$ vs $6 \%(\mathrm{p}<0.001)$ and $38 \%$ vs $27 \%$ $(p<0.001)$, respectively. All-cause mortality (11\% vs $9 \% ; p=0.306$ ) and hospitalisation rates ( $35 \%$ vs $29 \%$; $p=0.128$ ) did not differ between HF and NHF patients, respectively.

Conclusions Outcomes using the NICE approach of short waiting time targets for specialist assessment of patients with suspected HF and raised NTproBNP are not known. The model identifies an elderly population a high proportion of whom have HF. Irrespective of diagnosis, patients have high rates of adverse outcomes. These contemporary real-world data provide a platform for discussions with patients and shaping HF services.

\section{INTRODUCTION}

Heart failure (HF) is a common, chronic disease causing a significant public health and financial burden. Prevalence is estimated to be $1 \%-2 \%$ and increasing. ${ }^{12}$ Despite improvements in treatment the prognosis remains poor. Late diagnosis is common and increases the burden on patients and healthcare systems further. ${ }^{3}$ Early diagnosis and treatment represents an opportunity to improve morbidity and reduce hospitalisation and mortality, in particular for patients with HF with reduced ejection fraction.

Natriuretic peptide measurements have been established as the single most useful simple test to identify patients with possible HF and consequently are central in international diagnostic guidelines. ${ }^{4-7}$ Exactly how natriuretic peptides are effectively incorporated into systems of care is poorly defined. For example, optimal thresholds of natriuretic peptides and waiting time targets for specialist assessment for patients with suspected HF are unclear. Furthermore, in order to be useful in practice, guidelines often oversimplify complex clinical situations. Consequently, there is a lack of consensus between guidelines for evaluating patients when there is a clinical suspicion of HF.

In the UK, the National Institute for Health and Care Excellence (NICE) introduced guidelines and quality standards for the management of chronic HF to improve care. ${ }^{89}$ Natriuretic peptides and waiting time targets are central in these guidelines. It is recommended that patients with suspected $\mathrm{HF}$ and raised natriuretic peptides are referred for specialist HF assessment and echocardiography. Those with very high natriuretic peptides ( $\mathrm{N}$-terminal pro B-type natriuretic peptide, NTproBNP $>2000 \mathrm{pg} / \mathrm{mL}$ ) should be seen within 2 weeks and those with more modest elevation (NTproBNP $400-2000 \mathrm{pg} / \mathrm{mL}$ ) within 6 weeks. This waiting time-based recommendation is modelled on similar NICE referral pathways for suspected cancer and recognition that higher natriuretic peptide levels are associated with a worse prognosis. However, the natriuretic peptide thresholds and waiting time targets are arbitrary and as such this is an untested model. The population identified and associated patient outcomes using this approach have not been described but are important, both to evaluate the model and to shape future services. The aim of this study is to describe patient characteristics, HF diagnostic rates, and 1-year hospitalisation and mortality rates in a multicentre, real-world setting using this NICE model. 


\section{METHODS}

This is a multicentre, retrospective analysis of patients with suspected HF seen in dedicated HF clinics for specialist (HF consultant or a supervised senior HF trainee) assessment and echocardiography, in line with the contemporary NICE guidelines. ${ }^{8}$ Data were collected from two centres: Portsmouth Hospitals NHS Trust, a large district general hospital serving approximately 675000 patients, and University Hospital Southampton NHS Foundation Trust, a large university hospital serving approximately 500000 patients.

Consecutive patients with suspected $\mathrm{HF}$ and raised natriuretic peptides referred from primary care and seen in a specialist HF clinic from January 2014 to December 2016 (Portsmouth) and from December 2014 to December 2016 (Southampton) were included. One-stop HF clinics involve all patients undergoing an ECG and echocardiography by a cardiographer and cardiac physiologist, respectively, and specialist assessment by a physician, during a single visit, as previously described. ${ }^{10}$ The data period was covered by the 2010 version of the NICE guidelines. ${ }^{8}$ These guidelines recommend that patients with prior myocardial infarction (MI) do not require natriuretic peptide levels and should be seen within 2 weeks. Those without a history of MI are triaged according to natriuretic peptide level. Those with NTproBNP $>2000 \mathrm{pg} / \mathrm{mL}$ should be seen within 2 weeks (2-week pathway, 2WP) and those with NTproBNP $400-2000 \mathrm{pg} / \mathrm{mL}$ within 6 weeks (6-week pathway, 6WP).

Following clinical assessment, ECG and echocardiography, a diagnosis of HF was either confirmed or excluded by a HF specialist. HF was subdivided into HF with reduced ejection fraction (HFrEF) or HF with preserved ejection fraction (HFpEF) based on accepted definitions and the clinical decision of the HF specialist. Generally, HFrEF is defined as a left ventricular ejection fraction (LVEF) $\leq 40 \%$ or moderate or worse impairment of left ventricular systolic function, and HFpEF as LVEF $>40 \%$ or normal or mildly impaired left ventricular systolic function. Patients were investigated and managed in accordance with NICE and European Society of Cardiology (ESC) HF guidelines, individualised as appropriate. ${ }^{58}$ Patients with HFrEF routinely received ongoing follow-up from a multidisciplinary team, mainly from community HF specialist nurses supported by HF specialists. Those with HFpEF and those without HF (NHF) were usually discharged to primary care with a diagnosis and management plan where possible.

Data on patient characteristics, diagnosis and outcomes were collected from hospital coding data and electronic (and written if necessary) patient records. Outcome data included all-cause mortality and unplanned hospital admissions ( $\geq 1$ episode) in the 1-year period from the date of NTproBNP measurement. Hospitalisations were subdivided as cardiovascular, HF and noncardiovascular admissions according to hospital episode statistic data. Hospitalisations in each category were recorded as a binary outcome (hospitalisation vs no hospitalisation) regardless of the number of admissions per individual patient. Consequently, patients could be hospitalised a maximum of once in any single category but may have appeared in multiple hospitalisation categories. Patients in whom the diagnosis was unclear after assessment were included in the overall population analyses and in the $2 \mathrm{WP}$ versus $6 \mathrm{WP}$ analyses, but not in the HF versus NHF analyses.

A secondary analysis compared patients and outcomes between the participating centres.

The study was registered with the relevant clinical governance leads and audit departments at each centre. Written consent was not obtained as patient care was not affected. Some of the authors worked at both sites during the study period (AZ, GM). Only fully anonymised data were used in analyses.

\section{Statistical analyses}

Results are presented as median (IQR) and Kaplan-Meier survival curves. Continuous variables were tested using ShapiroWilk analysis to determine normality of distribution. Having determined that non-parametric tests were required, the groups were compared using Mann-Whitney $U$ test or $\chi^{2}$ test. Significance was determined if two-sided $\mathrm{p}$ values were $<0.05$.

\section{RESULTS}

\section{Patient population}

The study included 1271 patients referred to the specialist HF clinics: 1014 patients at Portsmouth and 257 at Southampton. Twenty-one additional patients with suspected HF and a history of MI but without NTproBNP levels were seen on the 2WP in the same time period but were not included.

Of the patients referred, 667 (53\%) were seen on the 2WP and $604(47 \%)$ on the 6WP. At Portsmouth, 91\% (2WP) and 96\% (6WP) of patients were offered an appointment within waiting time targets with mean waiting times of $11 \pm 6$ days and $36 \pm 20$ days, respectively. At Southampton, the equivalent data were $56 \%$ and $86 \%$ with waiting times of $20 \pm 28$ days and $29 \pm 24$ days, respectively. The baseline characteristics of the patients comparing the 2WP and 6WP groups are shown in table 1. Patients on the 2WP were slightly older than those on the 6WP

Table 1 Characteristics of patients referred with suspected heart failure using NICE guideline NTproBNP cut-offs according to target waiting time pathways

\begin{tabular}{|c|c|c|c|c|}
\hline & All patients $(\mathrm{N}=1271)$ & 2WP $(n=667)$ & $6 W P(n=604)$ & $P$ value \\
\hline Age (years) & $80(73-85)$ & $82(74-86)$ & 79 (73-84) & $<0.001$ \\
\hline Male & $591(47 \%)$ & $343(51 \%)$ & $248(41 \%)$ & $<0.001$ \\
\hline NTproBNP (pg/mL) & $1851(834-3633)$ & 3519 (2439-5729) & $875(621-1190)$ & $<0.001$ \\
\hline Haemoglobin (g/L) & $128(115-140)$ & $125(112-138)$ & $130(119-140)$ & $<0.001$ \\
\hline Creatinine $(\mu \mathrm{mol} / \mathrm{L})$ & $90(73-113)$ & $96(77-124)$ & $84(69-101)$ & $<0.001$ \\
\hline History of myocardial infarction & $190(15 \%)$ & $146(22 \%)$ & $44(7 \%)$ & $<0.001$ \\
\hline Atrial fibrillation & $641(51 \%)$ & $392(59 \%)$ & $249(42 \%)$ & $<0.001$ \\
\hline Hypertension & $833(66 \%)$ & $402(61 \%)$ & $431(72 \%)$ & $<0.001$ \\
\hline Diabetes mellitus & $272(21 \%)$ & $153(23 \%)$ & $119(20 \%)$ & 0.16 \\
\hline
\end{tabular}

Continuous data are presented as median (IQR).

$P$ values refer to comparisons between patients referred to 2WP and 6WP pathways.

NICE, National Institute for Health and Care Excellence; NTproBNP, N-terminal pro B-type natriuretic peptide ; 2WP, 2-week pathway; 6WP, 6-week pathway. 


\section{Entire Population}

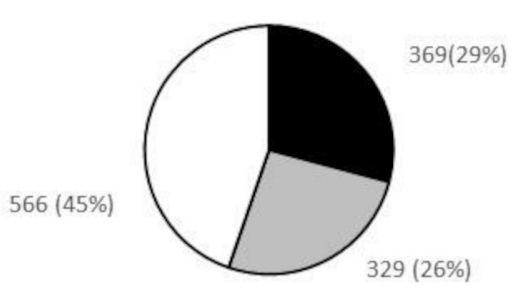

HFREF $\square$ HFPEF $\square$ NHF

2-week Pathway

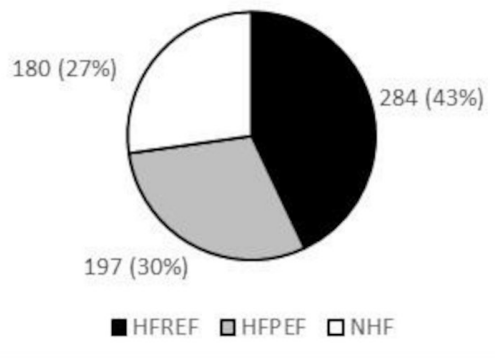

6-week Pathway

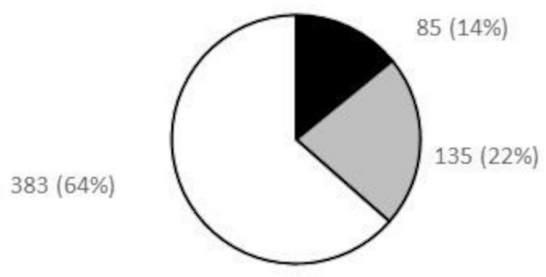

- $H F R E F \quad \square H F P E F \quad \square$ NHF

Figure 1 Diagnosis after specialist assessment in patients referred to the heart failure clinic. HFrEF, heart failure with reduced ejection fraction; HFpEF, heart failure with preserved ejection fraction; NHF, not heart failure.

and had higher rates of atrial fibrillation (AF) and previous $\mathrm{MI}$ (ie, many patients with a history of MI had NTproBNP checked in primary care regardless).

\section{HF diagnosis}

There were 698 (55\%) patients with confirmed HF diagnosis following specialist assessment: 369 (29\%) HFrEF and 329 (26\%) HFpEF. There were $566(45 \%)$ patients diagnosed as not HF (NHF). Within the 2WP group, $481(73 \%)$ patients had HF: 284 (43\%) HFrEF and 197 (30\%) HFpEF. Within the 6WP group, 217 (36\%) had HF: 85 (14\%) HFrEF and $132(22 \%)$ HFpEF (figure 1). It was unclear whether there was HF in 7 $(0.5 \%)$ patients.

The baseline characteristics of HF and NHF patient groups are shown in table 2. Patients in both HF and NHF groups were of similar ages. Patients in the HF group had much higher

Table 2 Patient characteristics according to heart failure diagnosis

\begin{tabular}{lccr}
\hline & HF $(\mathbf{n}=698)$ & NHF $(\mathbf{n}=566)$ & P value \\
\hline Age (years) & $81(74-86)$ & $79(73-85)$ & 0.025 \\
Male & $352(50 \%)$ & $234(41 \%)$ & 0.001 \\
NTproBNP $(\mathrm{pg} / \mathrm{mL})$ & $2815(1241-4890)$ & $988(633-2056)$ & $<0.001$ \\
\hline Haemoglobin $(\mathrm{g} / \mathrm{L})$ & $127(113-141)$ & $129(117-139)$ & 0.226 \\
Creatinine $(\mu \mathrm{mol} / \mathrm{L})$ & $93(75-116)$ & $87(71-106)$ & $<0.001$ \\
\hline History of myocardial infarction & $118(17 \%)$ & $72(13 \%)$ & 0.038 \\
Atrial fibrillation & $401(57 \%)$ & $237(42 \%)$ & $<0.001$ \\
\hline History of hypertension & $442(63 \%)$ & $390(69 \%)$ & 0.038 \\
History of diabetes mellitus & $171(25 \%)$ & $98(17 \%)$ & 0.002 \\
\hline
\end{tabular}

Continuous data are presented as median (IQR).

$P$ values refer to comparisons between patients with $\mathrm{HF}$ and NHF diagnoses.

$\mathrm{HF}$, heart failure; NHF, not heart failure; NTproBNP, N-terminal pro B-type natriuretic peptide.
NTproBNP levels (online supplementary table 1 by age groups), higher rates of previous MI, AF, diabetes and slightly higher baseline creatinine compared with the NHF group.

\section{Other diagnoses}

Within the NHF group, 483 (85\%) patients had their symptoms attributed to an alternative diagnosis following specialist assessment. The most common alternative diagnoses identified as the primary cause for symptoms and raised NTproBNP were AF (19\%), respiratory disease (18\%), hypertension (9\%), valvular heart disease (7\%) and ischaemic heart disease (4\%) (online supplementary table 2).

\section{Patient outcomes}

All-cause mortality at 1 year for the entire study population was $10 \%(n=129)$ and all-cause hospitalisation rate was $33 \%$ $(n=413)$ (table 3).

Both all-cause mortality and all-cause hospitalisation rates at 1 year from NTproBNP measurement were higher for those

Table 3 1-year all-cause mortality and hospitalisation rates for 2WP and 6WP pathways

\begin{tabular}{llccr}
\hline & $\begin{array}{l}\text { All } \\
(\mathrm{N}=1271)\end{array}$ & $\begin{array}{l}\text { 2WP } \\
(\mathbf{n}=667)\end{array}$ & $\begin{array}{l}\text { 6WP } \\
(\mathbf{n}=604)\end{array}$ & P value \\
\hline All-cause mortality & $129(10 \%)$ & $96(14 \%)$ & $33(6 \%)$ & $<0.001$ \\
\hline All-cause hospitalisation & $413(33 \%)$ & $252(38 \%)$ & $161(27 \%)$ & $<0.001$ \\
CV hospitalisation & $185(15 \%)$ & $135(20 \%)$ & $50(8 \%)$ & $<0.001$ \\
Non-CV hospitalisation & $302(24 \%)$ & $170(26 \%)$ & $132(22 \%)$ & 0.129 \\
\hline
\end{tabular}

Cardiovascular (CV) hospitalisation includes heart failure hospitalisation. Some patients were hospitalised in more than one category.

$P$ values refer to comparisons between $2 \mathrm{WP}$ and 6WP groups.

2WP, 2-week pathway; 6WP, 6-week pathway. 


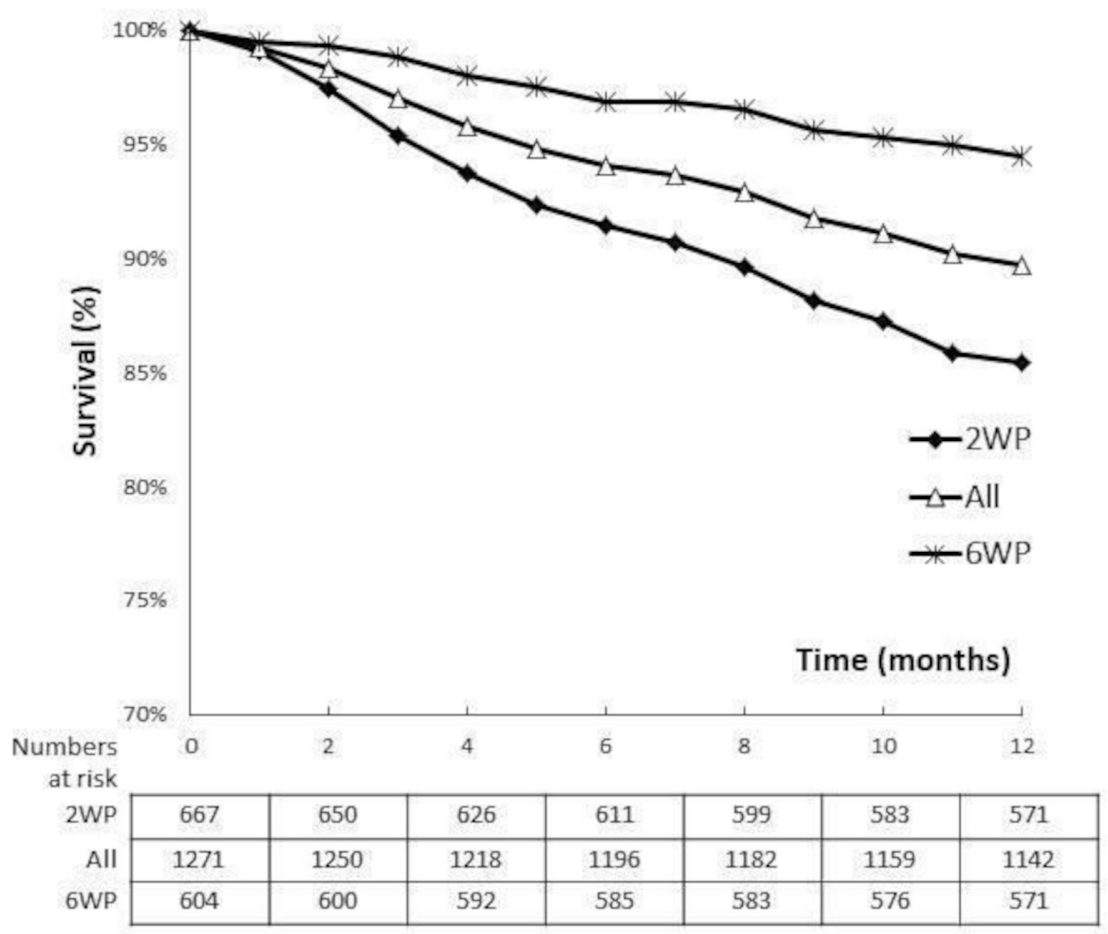

Figure 2 Survival curves for all patients (All) and patients referred on the 2WP and 6WP pathways. 2WP, 2-week pathway; 6WP, 6-week pathway.

patients on the 2WP compared with those on the 6WP (figure 2). The mortality rate was $14 \%(\mathrm{n}=96)$ vs $6 \%(\mathrm{n}=33)(\mathrm{p}<0.001)$ and hospitalisation rate $38 \%(\mathrm{n}=252)$ vs $27 \% \quad(\mathrm{n}=161)$ $(\mathrm{p}<0.001)$ for the 2WP vs 6WP patients, respectively (table 3 ).
There was no difference in either all-cause mortality or allcause hospitalisation rates in the HF group compared with the NHF group (figure 3$)$. The mortality rate was $11 \%(n=75)$ vs $9 \%$ $(\mathrm{n}=51)(\mathrm{p}=0.306)$ and hospitalisation rate was $35 \%(\mathrm{n}=247)$ vs

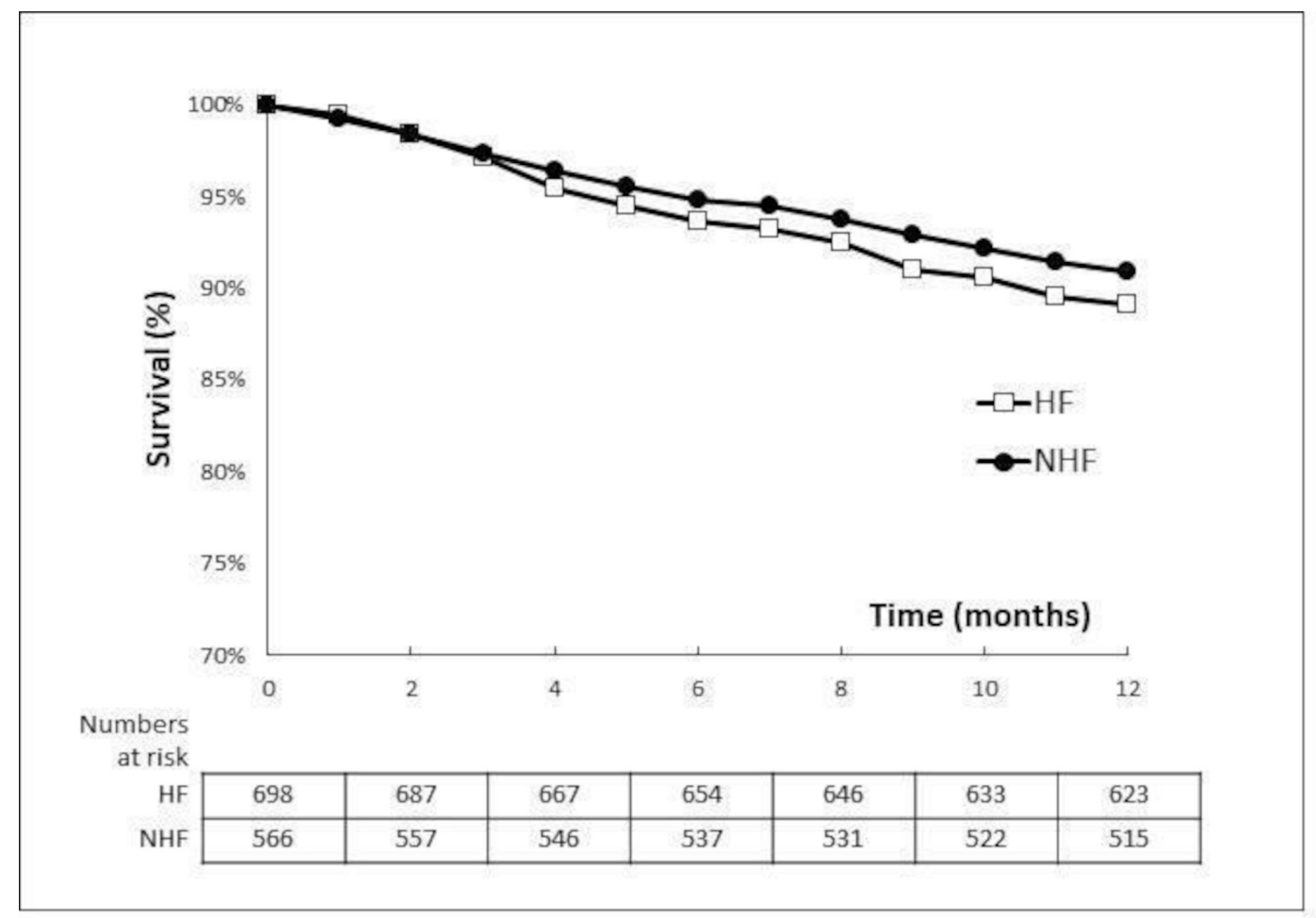

Figure 3 Survival curves for patients according to heart failure (HF) and not heart failure (NHF) diagnoses. 
Table 4 1-year all-cause mortality and hospitalisation rates according to diagnosis

\begin{tabular}{lccr}
\hline & HF $(\mathbf{n}=698)$ & NHF $(\mathbf{n}=566)$ & P value \\
\hline All-cause mortality & $75(11 \%)$ & $51(9 \%)$ & 0.306 \\
All-cause hospitalisation & $247(35 \%)$ & $163(29 \%)$ & 0.128 \\
CV hospitalisation & $130(19 \%)$ & $51(9 \%)$ & $<0.001$ \\
Non-CV hospitalisation & $166(24 \%)$ & $135(24 \%)$ & 0.978 \\
\hline
\end{tabular}

Cardiovascular (CV) hospitalisation includes HF hospitalisation. Some patients were hospitalised in more than one category.

$P$ values refer to comparisons between patients with heart failure (HF) and not heart failure (NHF) diagnoses.

$29 \%(\mathrm{n}=163)(\mathrm{p}=0.128)$ for the HF vs the NHF group, respectively (table 4).

\section{Secondary analysis}

Patient characteristics and outcomes were no different between the two recruiting centres, apart from a modest difference in the proportion of patients with $\mathrm{AF}$ (table 5).

\section{DISCUSSION}

\section{Principal findings and current literature}

Our study provides novel data describing the population of patients identified, and associated 1-year outcomes, when the NICE HF diagnostic pathways are applied in the real world. To date, there have been very limited data testing this pathway. Patients referred using the NICE model have a high incidence of HF when natriuretic peptides are very high (2WP). A much lower proportion of patients had HF in the presence of more modest elevations in natriuretic peptides (6WP). However, both pathways appear to compare favourably with the equivalent NICE 2WP for patients with suspected cancer, which results in a diagnosis of cancer in approximately $11 \%$ of patients. ${ }^{11}$ All patients had very high rates of hospitalisation and death at 1 year, regardless of whether the final diagnosis was HF or not, again particularly when natriuretic peptides were very high. Our findings therefore support using the approach of identifying and prioritising patients according to tiered natriuretic peptide thresholds. The findings reinforce the need for early recognition of potential HF in primary care with provision of natriuretic peptide testing. Identification of the highest risk patients is as important during the COVID-19 pandemic as ever before in order to risk-assess the urgency of patient assessment, prioritise appropriately, minimise harm to all patients and ensure efficient use of stretched healthcare services.

Table 5 Comparison of patient characteristics and outcomes between the two centres

\begin{tabular}{lccc}
\hline & $\begin{array}{l}\text { Portsmouth } \\
(\mathrm{n}=1014)\end{array}$ & $\begin{array}{l}\text { Southampton } \\
(\mathrm{n}=257)\end{array}$ & P value \\
\hline Age (years) & $80(74-86)$ & $80(73-85)$ & 0.210 \\
Male & $465(46 \%)$ & $125(49 \%)$ & 0.425 \\
NTproBNP (pg/mL) & $1827(835-3528)$ & $1916(819-4314)$ & 0.347 \\
History of myocardial infarction & $154(15 \%)$ & $36(14 \%)$ & 0.636 \\
Atrial fibrillation & $489(48 \%)$ & $152(59 \%)$ & 0.002 \\
History of hypertension & $666(66 \%)$ & $167(65 \%)$ & 0.833 \\
History of diabetes mellitus & $219(22 \%)$ & $53(21 \%)$ & 0.734 \\
1-year mortality & $99(10 \%)$ & $30(12 \%)$ & 0.365 \\
1-year hospitalisation & $324(32 \%)$ & $89(35 \%)$ & 0.413 \\
\hline P values rer to comparisons betwo
\end{tabular}

$P$ values refer to comparisons between Portsmouth and Southampton cohorts.

NTproBNP, N-terminal pro B-type natriuretic peptide .
A recent large UK study reported a 1-year mortality rate of approximately $19 \%$ for patients with newly diagnosed $\mathrm{HF}^{3}$ Their 1-year mortality rate is higher than our study. This may well reflect the fact that they included patients diagnosed with HF during hospital admission, who likely represent the severe end of the spectrum with the worst outcomes. ${ }^{12}$ The ESC HF long-term registry of 12440 patients reported a 1-year mortality of $6.4 \%$ in patients with chronic HF across 21 European countries, but did not include the UK. Their mortality rate was lower than our findings; however, the mean age of patients was only 65 years compared with our median of 81 years, and there was significant variability between the different European regions, ranging from $6.9 \%$ to $15.6 \% .^{13}$

There was no difference in either 1-year all-cause mortality or all-cause hospitalisation between patients diagnosed with HF versus those diagnosed as NHF. This was despite much higher median NTproBNP and higher baseline creatinine and rates of $\mathrm{AF}$, diabetes and previous $\mathrm{MI}$ in the HF group, which would all be expected to predict worse outcomes. ${ }^{14}$ This may reflect timely specialist assessment, investigation and implementation of evidence-based HF therapies (particularly for the HFrEF population), along with ongoing follow-up within a multidisciplinary

\section{Key messages}

What is already known on this subject?

- The National Institute for Health and Care Excellence (NICE) guidelines for the diagnosis and management of chronic heart failure (HF) recommend specialist assessment and echocardiography for patients with suspected HF on clinical grounds and elevated natriuretic peptides.

- Waiting time targets of 2 (NTproBNP $>2000 \mathrm{pg} / \mathrm{mL}$ ) or 6 (NTproBNP 400-2000 pg/mL) weeks are specified.

- The guidelines were introduced in 2010, modelled on similar NICE referral pathways for suspected cancer, in recognition of the poor outcomes in HF.

- Patient characteristics, HF diagnostic rates and outcomes using the NICE model in a real-world setting are not described.

What might this study add?

- Based on the recommended NICE model $53 \%$ of patients were referred on the 2-week pathway.

- Following specialist assessment $55 \%$ were diagnosed with $\mathrm{HF}$ ( $53 \%$ of those with HF with reduced ejection fraction).

- Patients with a new diagnosis of HF had a 1-year all-cause mortality of $11 \%$ and all-cause hospitalisation rate of $35 \%$.

- Patients on the 2-week pathway had worse outcomes than those on the 6-week pathway.

- There was no difference in outcomes between patients diagnosed with HF and those who did not have HF to explain their symptoms and elevated natriuretic peptides.

How might this impact on clinical practice?

- These novel data can improve clinician understanding of this large and important population and inform discussions with patients and their families.

- Understanding the outcomes of patients with suspected HF is necessary to structure and plan HF services at all times, and this includes during the COVID-19 pandemic, when service organisation, resource prioritisation and maintaining key nonCOVID-19 medical services are of crucial importance. 
team, improving outcomes in the HF group. It is also possible that studying a larger cohort would have identified a small difference between the groups.

Taylor $e t a l^{3}$ recently reported that patients with HF had a significantly worse prognosis than age, sex and practice matched controls. Our NHF cohort, however, were not controls but were patients with symptoms compatible with HF and elevated natriuretic peptides. While raised natriuretic peptides are known to associate with an adverse prognosis, outcomes for this specific patient group are not previously described ${ }^{14}$ In clinical practice it is tempting to reassure this cohort that they do not have HF. It is therefore important to note that their prognosis remains poor. This is despite the fact that HF specialists are also well placed to recognise and manage many of the alternative diagnoses in the NHF group, such as AF, hypertension or ischaemic heart disease. Moreover, unrecognised HF was unlikely to have been an important cause of this poor prognosis as subsequent hospitalisation due to development of HF in this cohort was only $2 \%$ at 1 year.

It is noteworthy that most hospitalisations were due to noncardiovascular causes, even in those with confirmed HF. Patients with HF are frequently and increasingly multimorbid; recent data suggest that at first presentation with HF patients have a mean of 5.4 important chronic comorbidities. ${ }^{2}$ Patients will therefore be at risk of hospitalisation due to competing non-cardiovascular causes and it is not surprising that these outnumber HF admissions. Non-cardiovascular hospitalisations will also sometimes include admissions for multisystem morbidity including HF but without HF being recorded. Finally, since HF causes multiorgan adverse effects, a proportion of the observed hospitalisations may have been due to sequelae of HF which are not obviously cardiovascular, such as renal impairment.

There is a substantial body of evidence describing improved outcomes for patients with HF when managed by specialist multidisciplinary teams. ${ }^{15} \mathrm{HF}$ is increasingly prevalent and our patient population is getting older and more complex. In the UK, it is estimated that the prevalence of patients living with HF increased by $23 \%$ between 2002 and 2014 and that there are approaching one million people with the diagnosis. ${ }^{2}$ Healthcare systems need to plan HF services appropriately to manage such a large and prevalent disease burden. Understanding the population and providing a well-structured multidisciplinary approach are key to improving services and quality of care.

\section{Strengths and limitations}

The main strength of this study is that it is a large, multicentre, real-world description of a cohort of consecutive patients with suspected HF managed according to the NICE pathways, which has not been previously described. Patient characteristics and outcomes were very similar between the two different centres, reducing the likelihood of centre-specific effects and suggesting that our findings are likely to be broadly generalisable.

The main limitation is that the data are retrospective and observational, which lend itself to potential unadjusted confounding factors and referral bias.

We only recorded admissions to the patient's local hospital and did not record recurrent admissions in the same admission category and therefore have underestimated the burden of hospitalisations. However, due to the organisation of care in the UK and the population of patients who have HF, the vast majority of patients with HF are very likely to have been admitted to the local hospital. It is also common in HF studies to censor patients from the analysis at the time of a first hospitalisation rather than record recurrent admissions. Furthermore, there is no reason to suspect that this would affect patients with HF or NHF disproportionately and therefore influence comparisons between the groups. Mortality is less likely to have been underestimated as primary care are informed of all registered patient deaths and electronic records integrated with secondary care.

Reported incident rates of new HF diagnoses suggest that perhaps more patients would have been identified given the size of the population served by the study sites. ${ }^{2}$ However, we have previously described relatively low referral rates from primary care to specialist services for patients with suspected HF. ${ }^{10}$ There was a significant difference in sample size between the two Trusts despite serving comparable patient populations. This is likely due to a combination of factors including the availability of directaccess echocardiography in primary care at Southampton and, to a lesser extent, a shorter recruitment period (2 vs 3 years). There was also a difference between centres in the proportion of patients seen within the waiting time targets. However, as the patient populations and outcomes were so similar between the two centres, these factors are unlikely to have had an important influence on the results.

The period of time studied meant patients were managed according to the 2010 NICE guidelines. ${ }^{8}$ These guidelines have since been simplified in 2018 such that a history of MI has been removed and all patients are simply triaged according to NTproBNP level. ${ }^{7}$ We only included patients with positive natriuretic peptides in our study. However, this resulted in the exclusion of $<2 \%$ of patients as most with a history of MI had NTproBNP measured regardless, so this is unlikely to have materially affected the results.

Finally, as with all retrospective data analyses involving health records, we relied on the accuracy of hospital episode statistic coding and medical records, which are relatively reliable but can at times be incomplete, unclear or inaccurate. A systematic review of UK discharge summary coding data reported a median accuracy of $90 \%$, while the Audit Commission reports an accuracy of $87 \%{ }^{16}$

\section{CONCLUSIONS}

Early identification of patients with HF represents an opportunity to improve patient morbidity and mortality. The optimal system of care to identify these patients is unknown and several different models have been proposed. Applying the UK NICE guidelines results in the identification of a high-risk elderly patient population. Following specialist review, approximately half of the patients have HF, and of those approximately half have HFrEF. Patients had high 1-year mortality and hospitalisation rates, regardless of whether they have HF. Approximately half of the patients had very high natriuretic peptides requiring rapid assessment (2WP). This group had a very high prevalence of HF and adverse outcomes. These contemporary real-world data can inform discussions with patients and their families and help shape HF services.

\section{Twitter Elena Cowan @ElenaCowan_}

Contributors GM defined the methods, acquired, analysed and interpreted the data, and drafted the manuscript. AZ acquired, analysed and interpreted the data and drafted the manuscript. EC analysed and interpreted the data and drafted the manuscript. LM, AF, PJC, KG, PH, RDA and PK helped acquire data and critically revised the manuscript. All authors read and approved the final manuscript.

Funding The authors have not declared a specific grant for this research from any funding agency in the public, commercial or not-for-profit sectors.

Competing interests None declared. 
Patient and public involvement Patients and the public were involved in the development of the NICE guidelines and quality standards. Patients or the public were not involved in the design, conduct or dissemination plans of our study.

Patient consent for publication Not required.

Ethics approval Formal ethical approval was not required in accordance with the UK Policy Framework for Health and Social Care Research guidance on quality evaluation projects which do not affect patient care.

Provenance and peer review Not commissioned; externally peer reviewed.

Data availability statement All data relevant to the study are included in the article or uploaded as supplementary information.

Open access This is an open access article distributed in accordance with the Creative Commons Attribution Non Commercial (CC BY-NC 4.0) license, which permits others to distribute, remix, adapt, build upon this work non-commercially, and license their derivative works on different terms, provided the original work is properly cited, appropriate credit is given, any changes made indicated, and the use is non-commercial. See: http://creativecommons.org/licenses/by-nc/4.0/.

\section{ORCID iDs}

Alice Zheng http://orcid.org/0000-0003-1902-4849

Elena Cowan http://orcid.org/0000-0003-0491-374X

Peter James Cowburn http://orcid.org/0000-0001-6210-2203

\section{REFERENCES}

1 Mosterd A, Hoes AW. Clinical epidemiology of heart failure. Heart 2007:93:1137-46.

2 Conrad N, Judge A, Tran J, et al. Temporal trends and patterns in heart failure incidence: a population-based study of 4 million individuals. Lancet 2018;391:572-80.

3 Taylor CJ, Ordóñez-Mena JM, Roalfe AK, et al. Trends in survival after a diagnosis of heart failure in the United Kingdom 2000-2017: population based cohort study. BMJ 2019:364:1223.
4 Mant J, Doust J, Roalfe A, et al. Systematic review and individual patient data meta-analysis of diagnosis of heart failure, with modelling of implications of different diagnostic strategies in primary care. In: NIHR health technology assessment programme: executive summaries. 13, 2009.

5 Ponikowski P, Voors AA, Anker SD, et al. 2016 ESC guidelines for the diagnosis and treatment of acute and chronic heart failure: the task force for the diagnosis and treatment of acute and chronic heart failure of the European Society of cardiology (ESC). Eur Heart J 2016:37:2129-200.

6 Yancy CW, Jessup M, Bozkurt B, et al. 2017 ACC/AHA/HFSA focused update of the 2013 ACCF/AHA guideline for the management of heart failure. J Am Coll Cardiol 2017;23:628-51.

7 Chronic heart failure in adults: diagnosis and management NICE guideline [NG106] 2018. Available: https://www.nice.org.uk/guidance/ng106

8 Chronic heart failure in adults: management, NICE Clinical guideline [CG108] 2010

9 NICE. Chronic heart failure in adults. Quality standard [QS9], 2011. Available: https:// www.nice.org.uk/guidance/qs9

10 Morton G, Philip L, Gilpin T, et al. Does specialist review for patients with suspected heart failure predict better outcomes? An observational study on the utility of compliance with NICE guidelines. BMJ Open 2018:8:e021856.

11 Meechan D, Gildea C, Hollingworth L, et al. Variation in use of the 2-week referral pathway for suspected cancer: a cross-sectional analysis. Br J Gen Pract 2012;62:e590-7.

12 Parenica J, Spinar J, Vitovec J, et al. Long-term survival following acute heart failure: the acute heart failure database main registry (AHEAD main). Eur J Intern Med 2013;24:151-60.

13 Crespo-Leiro MG, Anker SD, Maggioni AP, et al. European Society of cardiology heart failure long-term registry (ESC-HF-LT): 1-year follow-up outcomes and differences across regions. Eur J Heart Fail 2016:18:613-25.

14 Wang TJ, Larson MG, Levy D, et al. Plasma natriuretic peptide levels and the risk of cardiovascular events and death. N Engl J Med 2004;350:655-63.

15 Morton G, Masters J, Cowburn PJ. Multidisciplinary team approach to heart failure management. Heart 2018:104:1376-82.

16 Burns EM, Rigby E, Mamidanna R, et al. Systematic review of discharge coding accuracy. J Public Health 2012;34:138-48. 